\title{
Comparison between antegrade and retrograde cerebral perfusion or profound hypothermia as brain protection strategies during repair of type $\mathrm{A}$ aortic dissection
}

\author{
Sotiris C. Stamou ${ }^{1}$, Laura A. Rausch ${ }^{1}$, Nicholas T. Kouchoukos ${ }^{2}$, Kevin W. Lobdell ${ }^{3}$, Kamal Khabbaz ${ }^{4}$, \\ Edward Murphy ${ }^{5}$, Robert C. Hagberg ${ }^{6}$ \\ ${ }^{1}$ Department of Cardiothoracic Surgery, University of Iowa Hospitals and Clinics, Iowa City, IA, USA; ${ }^{2}$ Division of Cardiothoracic Surgery, \\ Missouri Baptist Medical Center, Saint Louis, MO, USA; ${ }^{3}$ Department of Thoracic and Cardiovascular Surgery, Sanger Heart and Vascular \\ Institute, Carolinas Medical Center, Charlotte, NC, USA; ${ }^{4}$ Division of Cardiac Surgery, Department of Surgery, Beth Israel Deaconess Medical \\ Center, Harvard Medical School, Boston, MA, USA; ${ }^{5}$ Thoracic and Cardiovascular Surgery, Spectrum Health, Fred and Lena Meijer Heart and \\ Vascular Institute, Grand Rapids, MI, USA; ${ }^{6}$ Department of Cardiac Surgery, Hartford Hospital, Hartford, CT, USA \\ Correspondence to: Sotiris C. Stamou, MD. Department of Cardiothoracic Surgery, University of Iowa Hospitals and Clinics, 200 Hawkins Drive, SE \\ 516 GH, Iowa City, IA 52242, USA. Email: cvsisfun@hotmail.com.
}

\begin{abstract}
Background: The goal of this study was to compare early postoperative outcomes and actuarial-free survival between patients who underwent repair of acute type A aortic dissection by the method of cerebral perfusion used.

Methods: A total of 324 patients from five academic medical centers underwent repair of acute type A aortic dissection between January 2000 and December 2010. Of those, antegrade cerebral perfusion (ACP) was used for 84 patients, retrograde cerebral perfusion (RCP) was used for 55 patients, and deep hypothermic circulatory arrest (DHCA) was used for 184 patients during repair. Major morbidity, operative mortality, and 5-year actuarial survival were compared between groups. Multivariate logistic regression was used to determine predictors of operative mortality and Cox Regression hazard ratios were calculated to determine the predictors of long term mortality.

Results: Operative mortality was not influenced by the type of cerebral protection (19\% for ACP, $14.5 \%$ for RCP and $19.1 \%$ for DHCA, $\mathrm{P}=0.729)$. In multivariable logistic regression analysis, hemodynamic instability [odds ratio $(\mathrm{OR})=19.6,95 \%$ confidence intervals $(\mathrm{CI}), 0.102-0.414, \mathrm{P}<0.001$ ] and $\mathrm{CPB}$ time $>200 \mathrm{~min}$ $(\mathrm{OR}=4.7,95 \% \mathrm{CI}, 1.962-1.072, \mathrm{P}=0.029)$ emerged as independent predictors of operative mortality. Actuarial 5-year survival was unchanged by cerebral protection modality (48.8\% for ACP, $61.8 \%$ for RCP and $66.8 \%$ for no cerebral protection, $\log$-rank $\mathrm{P}=0.844)$.

Conclusions: During surgical repair of type A aortic dissection, ACP, RCP or DHCA are safe strategies for cerebral protection in selected patients with type A aortic dissection.
\end{abstract}

Keywords: Aorta; aortic dissection; cerebral protection

Submitted Mar 13, 2016. Accepted for publication Apr 08, 2016

doi: $10.21037 /$ acs.2016.04.02

View this article at: http://dx.doi.org/10.21037/acs.2016.04.02

\section{Introduction}

Surgical repair of acute type A aortic dissections is associated with high mortality, which ranges from $2.8 \%$ to $30 \%$. This is often attributed to the emergent nature of the procedure, an elderly patient population, severe comorbidities, and oftentimes a large extent of aorta needing replacement (1-9). The necessity to clamp many of the large arteries supplying the brain during repair has consequently led to a heightened risk of permanent neurological damage (1). Damage to the brain is the most prevalent complication following aortic 
surgery (1-12). Attempts to mitigate this risk have evolved over time from the use of deep hypothermic circulatory arrest (DHCA) alone, to DHCA with retrograde cerebral perfusion (RCP), and finally to moderate hypothermic circulatory arrest (MHCA) with unilateral or bilateral antegrade cerebral perfusion (ACP) $(1,13)$.

We sought to evaluate the efficacy of cerebral protection strategies for patients following repair of acute type A aortic dissection. We compared the early and late outcomes between ACP, RCP and DHCA as neuroprotective strategies during repair of type A aortic dissection.

\section{Methods}

\section{Patients}

The Society of Thoracic Surgeons Databases at Beth Israel Deaconess, Carolinas Medical Center, Missouri Baptist Medical Center, Meijer Heart and Vascular Institute, and the University of Iowa Hospitals and Clinics were queried to identify all patients who underwent repair of aortic dissection between January 2000 and December 2010 and utilized either ACP, RCP or DHCA during surgery. A total of 324 patients underwent repair for acute type A aortic dissection. Of those, 84 were repaired using ACP, 55 had RCP and 184 had DHCA. Patients excluded were those that presented with a type A dissection who did not have surgery.

A preoperative diagnosis of aortic dissection was accomplished using computed tomography angiography (CTA) or transesophageal echocardiography (TEE). The diagnosis was later confirmed at the time of operation. A database was created for entry of demographic, procedural data and postoperative outcomes. These were prospectively entered by dedicated data-coordinating personnel. Long-term survival data were obtained from the Social Security Death Index (http://search.ancestry.com/search/ db.aspx? dbid=3693).

Prior to this analysis, study approval from the Institutional Review Boards of each center was obtained. Consistent with the Health Insurance Portability and Accountability Act of 1996 (HIPAA), patient confidentiality was consistently maintained.

\section{Definitions}

The Society of Thoracic Surgeons' national cardiac surgery database definitions were used for this study. Acute type A dissection was defined as any dissection that involved the ascending aorta with presentation within two weeks of the onset of symptoms. Previous cerebrovascular accident was defined as history of central neurologic deficit persisting for more than $24 \mathrm{~h}$. Chronic renal insufficiency was defined as a serum creatinine value $>2.0 \mathrm{mg} / \mathrm{dL}$. Diabetes was defined as a history of diabetes mellitus, regardless of duration of disease or need for oral agents or insulin. Recent myocardial infarction was defined as myocardial infarction occurring within 7 days. Depressed ejection fraction was defined as ejection fraction $<40 \%$. Hemodynamic instability was defined as hypotension (systolic blood pressure $<80 \mathrm{mmHg}$ ) or the presence of cardiac tamponade, shock, acute congestive heart failure, myocardial ischemia and or infarction. Prolonged ventilatory support was defined as pulmonary insufficiency requiring ventilatory support $>24$ hours, postoperatively. Postoperative stroke was defined as any new major (type I) neurologic deficit presenting inhospital and persisting $>72$ hours. Acute renal failure was defined as one or both of the following: (I) an increase in the serum creatinine to $>2.0 \mathrm{mg} / \mathrm{dL}$ and/or a $>2$-fold increase in the most recent preoperative creatinine level or (II) a new requirement for dialysis, postoperatively. Operative mortality included both (I) all deaths occurring during the hospitalization in which the operation was performed (even if death occurred after 30 days from the operation); and (II) those deaths occurring after discharge from the hospital, but within 30 days of the procedure.

\section{Operative technique}

The surgical approach was consistent irrespective of the type of cerebral protection used. The choice of cerebral protection strategy varied between surgeons but there were no significant differences in the strategy between hospitals. Intraoperatively, the diagnosis of type A aortic dissection was confirmed by TEE for all patients. A median sternotomy was created to provide access. Total cardiopulmonary bypass $(\mathrm{CPB})$ was provided by arterial cannulation of the femoral or right axillary artery and venous cannulation of the right atrium. Cold blood cardioplegia administration through an antegrade approach via the ostia of the coronary arteries and/or a retrograde approach through the coronary sinus was performed to ensure myocardial protection. If cerebral protection was indicated, it was initiated at this time through the use of either an antegrade or retrograde approach. ACP was initiated through the right axillary artery, while RCP was initiated through the superior 
vena cava. The right superior pulmonary vein provided access for vent placement in the left ventricle. Restoration of the aortic root was accomplished by resection of the intimal tear followed by repair or resuspension of the aortic valve and replacement of the ascending aorta. After reaching a mean cooling temperature range of 10 to $24{ }^{\circ} \mathrm{C}$ for DHCA or 25 to $32{ }^{\circ} \mathrm{C}$ for MHCA, the aortic clamp was removed and the aortic arch was examined. An arch replacement was performed when an arch tear was identified. The distal anastomosis was then completed and antegrade aortic perfusion was established. If the aortic valve and the sinuses were normal, resuspension of the aortic valve was performed by placing three polypropelene pledgeted sutures at the three valve commissures, along with replacement of the ascending aorta with a straight tube graft. If the aortic valve was structurally abnormal, but the sinuses were normal, aortic valve replacement with mechanical or tissue prosthesis and supracoronary aortic grafting were performed. If the aortic valve and sinuses were abnormal, from dilation (more than $5 \mathrm{~cm}$ ), or extension of the intimal tear to the valve, aortic root replacement (modified Bentall operation) with a tissue or mechanical valved-conduit was used. Teflon (polytetrafluoroethylene) strips were used to reinforce the proximal and distal anastomosis. In some patients, biological glue (BioGlue ${ }^{\circledR}$ surgical adhesive, Cryolife, Kennesaw, GA, USA) was used to better reapproximate the dissected layers.

\section{Data analysis}

\section{Univariate analysis}

Univariate comparisons of preoperative, operative, and postoperative variables were performed between patients repaired utilizing DHCA $(\mathrm{n}=184)$, ACP $(\mathrm{n}=84)$, and RCP $(\mathrm{n}=55)$. Normal distribution of continuous variables was assessed using the Kolmogorov-Smirnov test. Continuous variables were tested using either the one-way ANOVA test, while categorical variables were assessed by the chisquare or Fisher exact test, depending on the distribution of data. All tests were two-sided and a $\mathrm{P}$ value of $<0.05$ was considered statistically significant.

\section{Multivariable analysis}

A multivariable, stepwise, forward logistic regression analysis was conducted to determine independent predictors of operative mortality. The criterion for variable entry into the logistic model was a univariate probability level of
$\mathrm{P}<0.100$. The quality of the fit of the logistic model was tested with the Hosmer-Lemeshow goodness-of-fit test.

\section{Survival analysis}

Kaplan-Meier univariate unadjusted survival estimates were calculated and compared using a log-rank test for patients repaired utilizing ACP, RCP or DHCA. Cox Regression hazard ratios were calculated to determine the predictors of long term mortality. All analyses were conducted using SPSS statistical software Version 21 (IBM Corp, Armonk, NY, USA).

\section{Results}

\section{Preoperative characteristics}

Preoperative characteristics are summarized in Table 1. Only ejection fraction varied between the three groups $(\mathrm{P}=0.033)$. The Games Howell post-hoc test showed a higher ejection fraction in the RCP group compared to DHCA patients.

\section{Operative characteristics}

Operative characteristics of patients repaired utilizing ACP, RCP or DHCA, who underwent repair for acute type A aortic dissection are presented in Table 2. Patients repaired using ACP more frequently had prolonged $\mathrm{CPB}$ times (longer than 200 minutes), compared to patients who underwent DHCA or RCP $(\mathrm{P}=0.003)$. ACP patients had a longer average $\mathrm{CPB}$ time $(\mathrm{P}=0.023)$, in comparison with patients who underwent DHCA or RCP, as shown by the Bonferroni post-hoc test. Patients repaired using RCP had a longer average circulatory arrest time than those repaired with ACP or DHCA $(\mathrm{P}<0.001)$, with ACP having a longer average time compared to patients repaired under DHCA. A total arch replacement was required more frequently for patients repaired using ACP compared to patients repaired with DHCA or RCP $(\mathrm{P}<0.001)$. Circulatory arrest temperatures were lower in DHCA patients, while ACP patients had higher circulatory arrest temperatures $(\mathrm{P}=0.040)$. $\mathrm{RCP}$ was performed at a lower temperature than ACP.

\section{Postoperative characteristics}

Postoperative characteristics are depicted in Table 3. There was no difference in postoperative outcomes between patients who underwent ACP, RCP or DHCA during surgical repair. 


\begin{tabular}{|c|c|c|c|c|}
\hline Variable $^{a}$ & DHCA $(n=184)$ & $\operatorname{ACP}(n=84)$ & $\mathrm{RCP}(\mathrm{n}=55)$ & $P$ value \\
\hline Age (years) & 59 [19-83] & 58 [29-87] & 62 [23-83] & 0.314 \\
\hline Diabetes & $15(8.2 \%)$ & $6(7.1 \%)$ & $3(5.5 \%)$ & 0.794 \\
\hline Hypertension & $137(74.5 \%)$ & $67(79.8 \%)$ & $42(76.4 \%)$ & 0.639 \\
\hline Ejection fraction & 56 [20-75] & $58[30-75]$ & 59 [50-75] & 0.033 \\
\hline COPD & $14(7.6 \%)$ & $7(8.3 \%)$ & $4(7.3 \%)$ & 0.570 \\
\hline Creatinine & $1.3[0.6-3.8]$ & $1.2[0.5-3.9]$ & $1.1[0.4-2.4]$ & 0.313 \\
\hline Female gender & $59(32.1 \%)$ & $24(28.6 \%)$ & $17(30.9 \%)$ & 0.848 \\
\hline Arrhythmias & $20(10.9 \%)$ & 7 (8.3\%) & $6(10.9 \%)$ & 0.803 \\
\hline NYHA class & & & & 0.074 \\
\hline 1 & $17(9.2 \%)$ & $5(6.0 \%)$ & $4(7.3 \%)$ & - \\
\hline II & $43(23.4 \%)$ & $29(34.5 \%)$ & $22(40.0 \%$ & - \\
\hline III & $39(21.2 \%)$ & $16(19.0 \%)$ & $15(27.3 \%)$ & - \\
\hline IV & $85(46.2 \%)$ & $34(40.5 \%)$ & $14(25.5 \%)$ & - \\
\hline Cerebrovascular accident & $18(9.8 \%)$ & $4(4.8 \%)$ & $5(9.1 \%)$ & 0.378 \\
\hline Hemodynamic instability & $27(14.7 \%)$ & $12(14.3 \%)$ & $8(14.5 \%)$ & 0.997 \\
\hline$E F<40$ & $10(5.4 \%)$ & $4(4.8 \%)$ & 0 & 0.216 \\
\hline
\end{tabular}

${ }^{\text {a }}$ continuous data are shown as median (range) and categorical data are shown as percentage. ACP, antegrade cerebral perfusion; RCP, retrograde cerebral perfusion; COPD, chronic obstructive pulmonary disease; EF, ejection fraction; NYHA, New York Heart Association.

Table 2 Operative patient characteristics

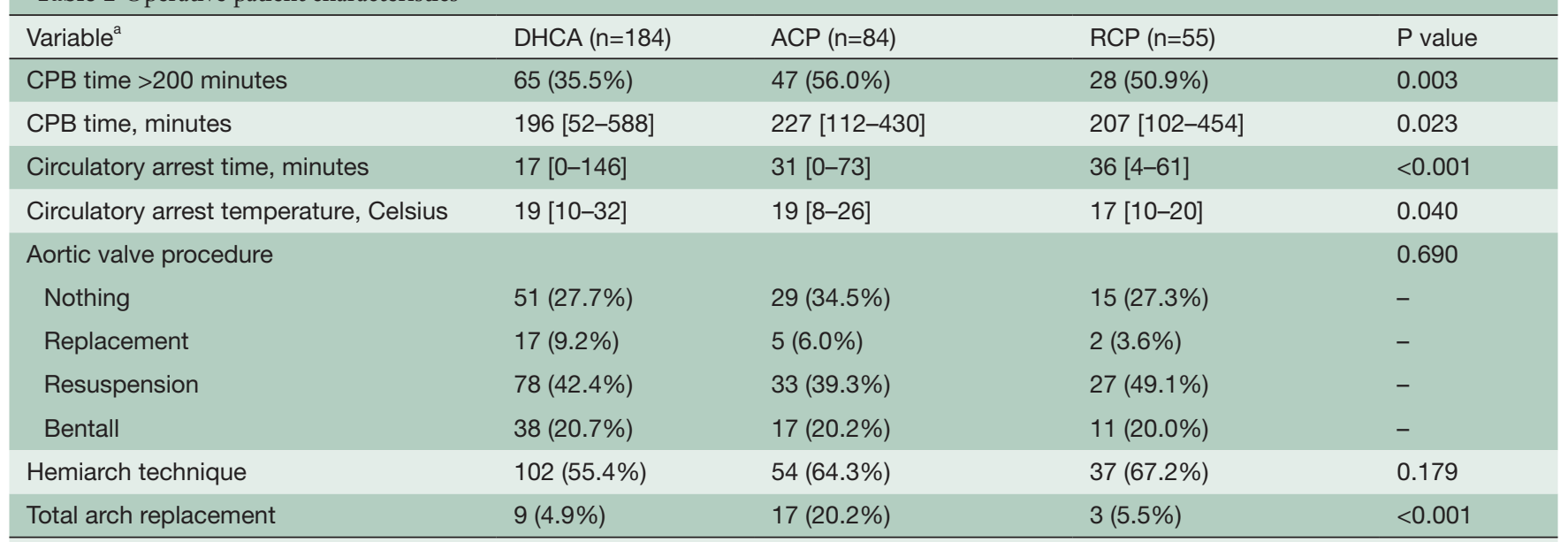

a , continuous data are shown as median (range) and categoric data are shown as percentage. ACP, antegrade cerebral perfusion; RCP, retrograde cerebral perfusion; CPB, cardiopulmonary bypass.

\section{Multivariate analysis}

In multivariable logistic regression analysis, hemodynamic instability [odds ratio (OR), 19.6, 95\% Confidence interval (CI), $0.102-0.414, \mathrm{P}<0.001]$ and $\mathrm{CPB}$ time $>200 \mathrm{~min}$ (OR, 4.7, 95\% CI, 1.962-1.072, $\mathrm{P}=0.029$ ) emerged as independent predictors of operative mortality.

\section{Survival analysis}

Actuarial Kaplan-Meier survival estimates are presented in the Figure 1. There was no difference in actuarial 5-year 


\begin{tabular}{|c|c|c|c|c|}
\hline Variable $^{a}$ & DHCA $(n=197)$ & $\operatorname{ACP}(n=112)$ & $\mathrm{RCP}(\mathrm{n}=55)$ & $P$ value \\
\hline Deep sternal wound infection & $4(2.2 \%)$ & $2(2.4 \%)$ & $3(5.5 \%)$ & 0.417 \\
\hline Acute renal failure & $38(20.7 \%)$ & $19(22.6 \%)$ & $8(14.4 \%)$ & 0.491 \\
\hline Hemodialysis & $18(9.8 \%)$ & $7(8.3 \%)$ & $1(1.8 \%)$ & 0.162 \\
\hline Stroke & $26(14.1 \%)$ & $12(14.3 \%)$ & $12(21.8 \%)$ & 0.361 \\
\hline Atrial fibrillation & $45(24.5 \%)$ & $10(11.9 \%)$ & $12(21.8 \%)$ & 0.062 \\
\hline Hospital length of stay (days) & 15 [0-99] & $14[1-61]$ & $16[5-86]$ & 0.753 \\
\hline Operative mortality & $35(19.1 \%)$ & $16(19 \%)$ & $8(14.5 \%)$ & 0.729 \\
\hline
\end{tabular}

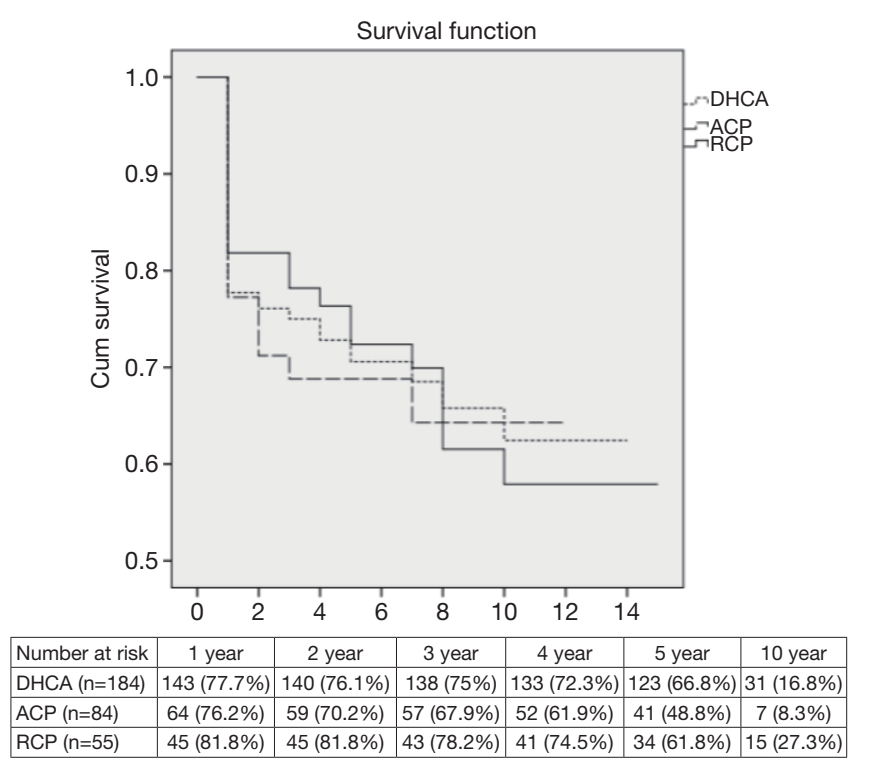

Figure 1 Actuarial 5-year survival curves for patients repaired using ACP, RCP or DHCA during acute type A aortic dissection repair. ACP, antegrade cerebral perfusion; RCP, retrograde cerebral perfusion; DHCA, deep hypothermic circulatory arrest.

survival for patients who underwent repair with ACP, RCP or DHCA (P value $=0.844$ ). Table 4 depicts the Cox regression hazard ratios for the predictors of late mortality.

\section{Discussion}

Our study compared early and late postoperative outcomes for patients who underwent repair of acute type A aortic dissection using ACP, RCP or DHCA. Patients who underwent surgery with $\mathrm{ACP}$ on average required more $\mathrm{CPB}$ time than those repaired with RCP or DHCA. Our results show that there is no difference in operative mortality for patients with type A aortic dissection repaired using ACP, RCP, or no cerebral protection. Hemodynamic instability and prolonged $\mathrm{CPB}$ times were independent predictors of operative mortality, regardless of cerebral protection strategy.

\section{Principal findings}

\section{Operative mortality}

There is a considerable debate over which cerebral perfusion method results in better brain protection and lower mortality. Our study found no difference in operative mortality with respect to ACP, RCP or DHCA during repair of acute type A aortic dissection. Other studies have also concluded that there is no difference in operative mortality between ACP and RCP (10,14-16).

While the method of cerebral perfusion may be a less important predictor of operative mortality, cerebral perfusion in general seems to be important for minimizing mortality. This seems to be less of an issue for shorter procedures where arrest times up to 30 to 40 minutes can be safely achieved with hypothermic circulatory arrest alone (2). However, Okita et al. reported significantly higher mortality for patients repaired with DHCA (14.5\%) compared to those repaired with RCP (3.4\%) (7). Similarly, Wiedemann et al. demonstrated higher operative mortality in patients repaired using DHCA alone (26\%) compared to those who have ACP $(13 \%)$ or RCP (16\%) (1). Some authors have concluded 


\begin{tabular}{llll}
\hline \multicolumn{2}{l}{ Table 4 Predictors of long term mortality } & & \\
\hline Variable & HR & $95 \% \mathrm{Cl}$ & P value \\
\hline Age & 19.7 & $1.020-1.054$ & 0.000 \\
\hline Hemodynamic instability & 9.4 & $0.301-0.768$ & 0.002 \\
Postoperative stroke & 10.5 & $0.302-0.745$ & 0.001 \\
\hline Postoperative renal failure & 9.1 & $0.344-0.797$ & 0.003 \\
Cerebral perfusion $>200$ min & 10.8 & $0.351-0.768$ & 0.001 \\
\hline HR, hazards ratio; $\mathrm{Cl}$, confidence interval. & & &
\end{tabular}

that this particularly happens when circulatory arrest more than 60 minutes, regardless of cerebral protection. When circulatory arrest time is greater than $60 \mathrm{~min}$, there is a twofold increase in the risk of mortality (3).

Our study documented a higher mortality with $\mathrm{CPB}$ time of greater than 200 minutes (OR=4.7, 95\% CI, 1.962-1.072, $\mathrm{P}=0.029)$. This represents patients who required more complex aortic reconstruction such as total arch replacement. More complex reconstructions were more frequently performed using ACP, as reported in previous studies (4). In our study, ACP was associated with a longer CPB time, which has also been supported in the literature (5-7). The use of ACP for longer surgeries has been shown to minimize global ischemia and extend the safe HCA time $(8,9)$. When a complex aortic reconstruction was anticipated, surgeons preferred ACP to RCP or DHCA, similar to previous reports $(8,9)$. In contrast, RCP was the preferred strategy when shorter $(<30 \mathrm{~min})$ circulatory arrest times are required and when the brain vessels were atheromatous, as a means of reducing the risk of embolic stroke $(8,10,11)$. Patients repaired using ACP underwent significantly more complex procedures such as total arch replacement which inherently required longer CPB times. Despite this disadvantage, ACP demonstrated the same operative mortality as RCP. With the appropriate selection of cerebral perfusion employed, depending on the type of procedure undertaken, we believe that either ACP or RCP can be effective.

\section{Cerebral protection strategy and risk of stroke}

The incidence of stroke $(\mathrm{P}=0.361)$ did not differ for patients who underwent surgery for type A aortic dissection with ACP, RCP or DHCA. RCP patients demonstrated a trend towards a higher rate of postoperative stroke that may be attributed to the fact that more patients in the RCP group had a history of cerebrovascular accident. Other studies have also demonstrated no difference between RCP and ACP for postoperative stroke; however, RCP tended to have more cases of transient neurological damage $(4,6,12,14)$. The most important risk factors for stroke are circulatory arrest time over 40 minutes and prolonged CPB time (15). While there was a difference in circulatory arrest times in our study, most notably between DHCA patients (17 min, mean) compared to ACP (31 min, mean) and RCP (36 min, mean), the majority of patients had circulatory arrest times under 40 minutes. Given that there was no difference in the incidence of stroke with all perfusion strategies maintained in a relative range of safety, our data suggests that cerebral perfusion technique alone does not impact the risk of stroke.

\section{Actuarial survival}

Most independent predictors of long-term mortality following aortic dissection repair have been attributed to cardiovascular causes such as stroke, myocardial infarction and congestive heart failure. However, at least $50 \%$ of deaths following discharge have been attributed to other comorbid conditions and not directly related to the repaired aorta (16). These include older age at presentation, chronic renal failure and hemodynamic instability as significant predictors of long-term mortality (16-19). Similar to the literature, our study found age at time of surgery, hemodynamic instability, postoperative stroke, renal failure and CPB time greater than $200 \mathrm{~min}$ to be independent predictors of late mortality (Table 4). However, our study found no difference in 5-year survival rates for patients repaired using ACP, RCP or DHCA (Figure 1). This corresponds well with the fact that we didn't see any major impact on comorbidities by perfusion technique in the perioperative period as well. Without any major incidence in morbidities associated with method of perfusion strategy, we believe that long-term survival is minimally impacted by cerebral perfusion technique.

\section{Clinical implications}

We conducted a multi-institutional observational study 
to assess the impact of methods of cerebral protection on operative characteristics and short- and long-term outcomes following repair of acute type A aortic dissection. In our study we examined an unselected cohort of patients from five academic institutions. This study compared patients that underwent surgery for type A aortic dissection with DHCA, ACP or RCP and evaluated the outcomes and operative mortality. Operative mortality was not statistically significant and was not shown to differ between the different forms of cerebral protection. The choice of whether to utilize RCP or ACP during hypothermic circulatory arrest is dependent on the extent of the aortic dissection and the patient. Currently, the use of ACP via right axillary cannulation with moderate hypothermia for complex aortic reconstructions is preferable in complex aortic reconstructions, since (I) it allows prevention of cerebral ischemia; (II) can be initiated before the chest is open; (III) provides safe and almost unlimited length in time for cerebral protection; and (IV) the axillary level is less likely to be the site of atheromatous lesions or dissection $(4,8,11,20-22)$. When the expected circulatory arrest time is relatively brief (less than 30 minutes), RCP or DHCA are safe alternative neuroprotective strategies during repair of type A dissection $(4,8,14,23,24)$.

\section{Study limitations}

Inherent limitations of a retrospective multi-institution investigation inevitably affected our study. Bias may have also been introduced into the analysis since different surgeons from five different institutions performed the procedures. Selection bias may affect the results and it may be possible that the results regarding each neuroprotection strategy may reflect the results of the surgeon performing the operation. Although the duration of cerebral perfusion time is not provided in our study, the duration of cerebral perfusion was almost equivalent to the duration of circulatory arrest time for the ACP and RCP groups. The small sample size was another limitation of our study that did not allow propensity score matching between the three groups to identify differences. Further study of reoperations on the remaining dissected aorta, the differences in outcomes based on use of unilateral or bilateral ACP and the fate of the false lumen were outside the scope of our analysis. In future, these should be the focus for evaluating long-term outcomes of acute type A aortic dissection repair.

\section{Conclusions}

The use of cerebral protection during surgical repair of type A aortic dissection should be evaluated on a case-bycase basis dependent on the preoperative patient conditions, complications that occur during surgery and surgical procedures performed. Our findings suggest that during surgical repair of type A aortic dissection, ACP, RCP or DHCA have acceptable morbidity and mortality in selected patients. The presence of hemodynamic instability prior to surgery and prolonged CPB time increases the risk of early and late mortality regardless of cerebral protection strategy.

\section{Acknowledgements}

None.

\section{Footnote}

Conflicts of Interest: The authors have no conflicts of interest to declare.

Ethical Statement: The study was approved by the Institutional Review Boards of each center. Consistent with the Health Insurance Portability and Accountability Act of 1996 (HIPAA), patient confidentiality was consistently maintained.

\section{References}

1. Wiedemann D, Kocher A, Dorfmeister M, et al. Effect of cerebral protection strategy on outcome of patients with Stanford type A aortic dissection. J Thorac Cardiovasc Surg 2013;146:647-55.

2. Bachet J. What is the best method for brain protection in surgery of the aortic arch? Selective antegrade cerebral perfusion. Cardiol Clin 2010;28:389-401.

3. Krüger T, Weigang E, Hoffmann I, et al. Cerebral protection during surgery for acute aortic dissection type A: results of the German Registry for Acute Aortic Dissection Type A (GERAADA). Circulation 2011;124:434-43.

4. Bavaria JE, Brinster DR, Gorman RC, et al. Advances in the treatment of acute type A dissection: an integrated approach. Ann Thorac Surg 2002;74:S1848-52; discussion S1857-63.

5. Okita Y, Takamoto S, Ando M, et al. Mortality and cerebral outcome in patients who underwent aortic arch operations using deep hypothermic circulatory arrest with 
retrograde cerebral perfusion: no relation of early death, stroke, and delirium to the duration of circulatory arrest. J Thorac Cardiovasc Surg 1998;115:129-38.

6. Usui A, Miyata H, Ueda Y, et al. Risk-adjusted and casematched comparative study between antegrade and retrograde cerebral perfusion during aortic arch surgery: based on the Japan Adult Cardiovascular Surgery Database : the Japan Cardiovascular Surgery Database Organization. Gen Thorac Cardiovasc Surg 2012;60:132-9.

7. Usui A, Yasuura K, Watanabe T, et al. Comparative clinical study between retrograde cerebral perfusion and selective cerebral perfusion in surgery for acute type A aortic dissection. Eur J Cardiothorac Surg 1999;15:571-8.

8. Rylski B, Urbanski PP, Siepe M, et al. Operative techniques in patients with type A dissection complicated by cerebral malperfusion. Eur J Cardiothorac Surg 2014;46:156-66.

9. Aebert H, Reber D, Kobuch R, et al. Aortic arch surgery using moderate systemic hypothermia and antegrade cerebral perfusion via the right subclavian artery. Thorac Cardiovasc Surg 2001;49:283-6.

10. Ueda Y. What is the best method for brain protection in surgery of the aortic arch? Retrograde cerebral perfusion. Cardiol Clin 2010;28:371-9.

11. Svensson LG, Nadolny EM, Penney DL, et al. Prospective randomized neurocognitive and S-100 study of hypothermic circulatory arrest, retrograde brain perfusion, and antegrade brain perfusion for aortic arch operations. Ann Thorac Surg 2001;71:1905-12.

12. Okita Y, Minatoya K, Tagusari O, et al. Prospective comparative study of brain protection in total aortic arch replacement: deep hypothermic circulatory arrest with retrograde cerebral perfusion or selective antegrade cerebral perfusion. Ann Thorac Surg 2001;72:72-9.

13. Misfeld M, Leontyev S, Borger MA, et al. What is the best strategy for brain protection in patients undergoing aortic arch surgery? A single center experience of 636 patients. Ann Thorac Surg 2012;93:1502-8.

14. Bavaria JE, Pochettino A, Brinster DR, et al. New paradigms and improved results for the surgical treatment of acute type A dissection. Ann Surg 2001;234:336-42; discussion 342-3.

15. Svensson LG, Crawford ES, Hess KR, et al. Deep hypothermia with circulatory arrest. Determinants of stroke and early mortality in 656 patients. J Thorac Cardiovasc Surg 1993;106:19-28; discussion 28-31.

16. Tsai TT, Evangelista A, Nienaber CA, et al. Long- term survival in patients presenting with type A acute aortic dissection: insights from the International Registry of Acute Aortic Dissection (IRAD). Circulation 2006;114:I350-6.

17. Glower DD, Speier RH, White WD, et al. Management and long-term outcome of aortic dissection. Ann Surg 1991;214:31-41.

18. Svensson LG, Crawford ES, Hess KR, et al. Dissection of the aorta and dissecting aortic aneurysms. Improving early and long-term surgical results. Circulation 1990;82:IV24-38.

19. Haverich A, Miller DC, Scott WC, et al. Acute and chronic aortic dissections--determinants of longterm outcome for operative survivors. Circulation 1985;72:II22-34.

20. Ogino H, Sasaki H, Minatoya K, et al. Evolving arch surgery using integrated antegrade selective cerebral perfusion: impact of axillary artery perfusion. J Thorac Cardiovasc Surg 2008;136:641-8; discussion 948-9.

21. Halkos ME, Kerendi F, Myung R, et al. Selective antegrade cerebral perfusion via right axillary artery cannulation reduces morbidity and mortality after proximal aortic surgery. J Thorac Cardiovasc Surg 2009;138:1081-9.

22. Immer FF, Moser B, Krähenbühl ES, et al. Arterial access through the right subclavian artery in surgery of the aortic arch improves neurologic outcome and mid-term quality of life. Ann Thorac Surg 2008;85:1614-8; discussion 1618.

23. Bavaria JE, Pochettino A. Retrograde cerebral perfusion (RCP) in aortic arch surgery: efficacy and possible mechanisms of brain protection. Semin Thorac Cardiovasc Surg 1997;9:222-32.

24. Appoo JJ, Augoustides JG, Pochettino A, et al. Perioperative outcome in adults undergoing elective deep hypothermic circulatory arrest with retrograde cerebral perfusion in proximal aortic arch repair: evaluation of protocol-based care. J Cardiothorac Vasc Anesth 2006;20:3-7.

Cite this article as: Stamou SC, Rausch LA, Kouchoukos NT, Lobdell KW, Khabbaz K, Murphy E, Hagberg RC. Comparison between antegrade and retrograde cerebral perfusion or profound hypothermia as brain protection strategies during repair of type A aortic dissection. J Thorac Dis 2016;5(4):328335. doi: 10.21037/acs.2016.04.02 